\title{
Interdisciplinary Collaboration for Investigating Medication Errors Causes: Six Sigma Methodologies
}

\author{
Fatma M. Elnady Abd Rabou ${ }^{1}$, Hanaa Azmi Saad ${ }^{2}$, \\ Sanaa Mohamed Abou El Ella ${ }^{3}$ \\ ${ }^{l}$ (Lecturer of Nursing Administration, Faculty of Nursing, Fayoum University) \\ ${ }^{2}$ (Lecturer of Nursing Administration, Faculty of Nursing Modern University for Technology and Information) \\ ${ }^{3}$ (CEO of Es-Salam Hospital)
}

\begin{abstract}
:
Background: Organizations which engaged in continuous improvement to improve outcomes had certain key factors such as having a quality-centered culture, and to overcome barriers directly involve top and middlelevels leaders, integrating improvement efforts with organizational priorities, and actively developing interdisciplinary teams for continuous improvement.

Aim: Study aimed to (1) assess hospital team efforts in tracing medication errors causes using incident report and (2) investigate medication errors causes through using six sigma methodologies among nursing staff.

Subject and Methods: A retrospective descriptive research design was used; study setting was in As-Salam international Hospital. The study was conducted by retrospectively auditing of total hospital incident report ( $n$. 679). Two tools were used for collecting data: (1) medication incident report and (2) quality improvement tools as brainstorming technique, process map, Pareto chart test, and cause and effect diagram.

Results: The study results showed that, regarding to the overall causes, medication administration errors was the highest percentage among all causes (52\%), and according to pareto test missed dose cause was the highest percentage of medication administration errors $(60.4 \%)$, while root causes of medication administration errors revealed that the process itself as well as the human factors were the main root causes.

Conclusion: The current study had accepted the research hypothesis by evidence that, interdisciplinary team by using Six sigma can identify the root cause of medication administration errors inside the hospital which is missed dose in addition to the process itself as well as the human factors were the major causes. Recommendation: It is recommended that, quality improvement advancing program and peer review should be implemented to assist in identifying causes of errors and eradicating its recurrence. All managerial categories should be engaged in the improvement process to get their commitment to quality standards. Staff nurses educate about medication administration should be conducted. Training on improvement process should be provided to increase awareness and force the internal auditing at unit level.
\end{abstract}

Keywords: Interdisciplinary team, Incident report, Six Sigma, Medication errors.

\section{Introduction}

Priorities for healthcare managers are to engage in establishing goals and strategies to improve the quality of care, they get involved in setting the quality agenda, to support quality improvement, promote a safety culture, cultivate leaders, manage resisters, plan ahead to procure organizational resources for quality. (Parand et al., 2014)

Succession in healthcare organization depends primarily on the partnership between members of the health team; this collaboration is now characterized by a culture which leads to provide comprehensive services. Collaboration between health care professionals provides a way to address the complexities inherent in health care settings. Interdisciplinary collaboration allows health teams from multiple disciplines with shared objectives, decision-making responsibilities, and power to work together to offer quality patient care (Bender, Connelly, \& Brown, 2013).

Errors in healthcare result in part from poorly designed complex systems. Determination of medication errors causes should be couple with assessment of the severity of the error. Quality management processes should include programs to decrease the incidence of all medication errors, effort should be concentrated on eliminating the causes associated with greater levels of severity. They should establish mechanisms for tracking drugs or drug classes that are involved in medication errors (Taghizadegan, 2011).

Medication error is any avoidable event that leads to inappropriate medication use or patient harm while the medication is in the control of the healthcare professional or patient. Such events may be related to professional practice, healthcare products, procedures, and systems, including prescribing, order communication, product labeling, packaging, compounding, dispensing, distribution, administration, education, 
monitoring, and use. (National Coordinating Council for Medication Error Reporting and Prevention 2015).

Bush (2010), who stated that, medication errors explained how a multidisciplinary team collected completed reporting forms, assigned severity and likelihood of recurrence scores, and then entered the information into a central reporting system to give feedback about the potential causes and find out the proper solution, one more way the team can perform was traced how a reported incident was investigated, interviewing with those who were involved in each incident, and subsequently met to discuss and agree on findings. Incident reporting system is a central notification and recording of incidents that led to patient harm, or could have caused harm, it fundamentally consists of a multistage process, starting with detection, analysis, learning and finally feedback, the majority of the studies have focused on the first stage of reporting, while investigating reports thoroughly and involving multiple stakeholder input can enhance the value of the system (Hewitt, 2013).

Six Sigma methodologies provide the hospitals a new dynamic approach to solve the problems and thus leading them to the peak of success. The success story of many big and small healthcare organizations who adopted the six sigma methodology has become a guiding plan to replace their old methodology by the same to achieve the acme of success (Taghizadegan, 2011). It can reduce variability and waste by translating to fewer errors, better processes, improved patient care, greater patient satisfaction rates, and happier, more productive employees. To achieve these goals, the DMAIC (Define-Measure-Analyze-Improve-Control) must be implementing (Bisgaard, 2014).

According to Esmail, (2011) the five phases in the DMAIC model has several tools that can be used in each phase, root cause or fishbone analysis is one of the tool that organizations can use to evaluate practice, risk and mistakes when they occur. It assists teams in categorizing the many potential causes of problems or issue in an orderly ways and in identifying the root causes from its origin in addition to generating solution for the possible reasons why a process is beginning to have difficulties. In the analyze phase, improvement team (multidisciplinary team) sought to discover root cause of the major contributors to the problem. Suggestions were generated by means of brainstorming. The resulting list is organized by means of cause-effect diagrams so the team could discern the specific assumptions of root causes that can be tested and causes identified (Jusko, 2012).

Significance of the study:

In an attempt to ensure patients' safety in an organization, numerous efforts were carried out to identify the most important problems which pose a serious threat to the patients and affect hospital reputation. Interdisciplinary team was formed to elicit a list of problems; the first priorities were medication errors. The effort was made to create an environment for improving productivity and efficiency that would decrease errors and prevent the occurrence. Assuring quality of care at hospital, Interdisciplinary team had embraced of Six Sigma methodology to improve medication errors.

Aim of the study:

This study aims to:

1. Assess hospital team efforts in tracing medication errors causes using incident report

2. Investigate medication errors causes through using six sigma methodologies among nursing staff.

\section{Research design:}

\section{Subjects And Methods:}

Retrospective descriptive research design was used in this study.

Research hypothesis:

Whenever there is close interdisciplinary collaboration between the relevant departments to study and investigate the possible medication errors causes, the greater the chance of successful implementation and improvement probability will take place.

\section{Research settings:}

The study was carried out in As-Salam International Hospital (total bed is 215) which includes multi-specialty department as (Emergency, medical imaging, oncology, rehabilitation, neurology, internal medicine, cardiac, obstetric, surgical, urology department as well as five intensive care units, operating theater, medical and surgical floors.

Sample:

\section{Medication Incident Report;}

The study sample was retrospectively auditing overall medication incident report (n. 679) from the period of October to December, 2014; it was taken as a sample. 
Tools for data collection:

1. The First Tool:

Medication error Incident Report:

Researchers' initial focus has been on measuring errors through using incident report for problems identification.

\section{The Second Tool:}

\section{Quality Improvement Tools:}

To get clear idea about the research problem and feasibility of the study the following tools were used as brainstorming technique, process map, weighted decision matrix medication incident data were measured and analyzed by using Pareto chart test, cause and effect diagram (fish bone diagram) and multi voting technique.

\section{Field Work/Procedure: \\ Preparatory Phase:}

Steering committee is consisted of members of upper management and heads of functional departments. This committee appointed employees with relevant daily floor level experience in various associated processes as members of the multidisciplinary health team. This group of eight members includes: quality manger, medical director specialist physician manger, internal medicine physician, one physician of the surgical department, chief nursing officer, medical floors' nurse supervisor, and surgical floors' nurse supervisor, the team received a special training. The group had enrolled in a training course related to six sigma methodologies and became the leaders by the hospital quality deportment to direct teams in project execution.

\section{Implementation Phase:}

The data was collected from the beginning of October 2014 and was completed by the end of December 2014; the field work was started by obtaining an official permission from Chief Executive Officer of As-Salam International Hospital. The six Sigma methodologies which consist of five phases define, measure, analysis, improve and control (DMAIC) but because the aim of the study was investigating medication administration errors causes, only define measure phases were used to cover the subject.

\section{Phase I: "Define"}

The "Define" phase consisted generally of identify integral elements of a process such as individuals involved and steps required. The multidisciplinary team subsequently first identified the following errors, based on Joint Commission International (JCI) standards. Firstly the operational description of missed dose, delay administration, wrong frequency, and wrong administration was made. Subsequently, the problem identified by the team that the medication error rate reported was high in the hospital to many interruptions and challenges faced drug administration process resulting in work overload which in turn affecting the patients safety of care delivered.

\section{Second Phase: "Measure the Problem"}

1. In measure phase, types and number of errors that occurred during the process and its Sigma rating were collected as baseline performance. The team created a process map for medication management process aiming to review and verify the process maps, the process of medication administration involves four steps, administration ordering, prescribing, handling and dispensing. Next step was auditing medication incident report to estimate rate by computed from the ratios of the total number of reported incident and the total numbers of errors are profiled on medication administration record by nurses then records errors by type and who committed them.

2. Sorting of errors during the measure phase was arranged in descending order to create a Pareto Chart. A Pareto chart is used to highlight the most common source of defects or the most frequently seen defect out of data collected. The Pareto test diagram showed that majority of the errors occurred during medication administration process steps. During the measure phase, Fish bone analysis or Root Cause and Process Flow Analysis were used which determined the various interruptions and challenges faced during medication administration.

3. Once the baseline performance defined, the "measurement" phase determined areas of high variability and identified potential causes. In this step, process map diagrams demonstrated areas of greatest process inconsistency or lack of role clarity that require more immediate intervention. In this stage the multidisciplinary health team was generated scoring system for testing all potential root causes to determine the higher two values for each category by using multi-voting technique.

4. Finally the multidisciplinary health team generated weighting data scale for additional testing of potential root causes of medication administration errors by using weight ranged from 5 to 1 from high relation to less one's. After the subsequent investigation, the multidisciplinary team using a multi voting technique to 
determine the highest score of possible root cause (missed dose) from the different types of errors followed by extra testing to summarize the medication administration errors.

\section{Ethical Consideration:}

An official letter contained clarifying the title, purpose\& setting of the study was send and approval was obtained from hospital administrators, all the ethical concepts were considered such as respect of truth, anonymity and confidentiality.

Result

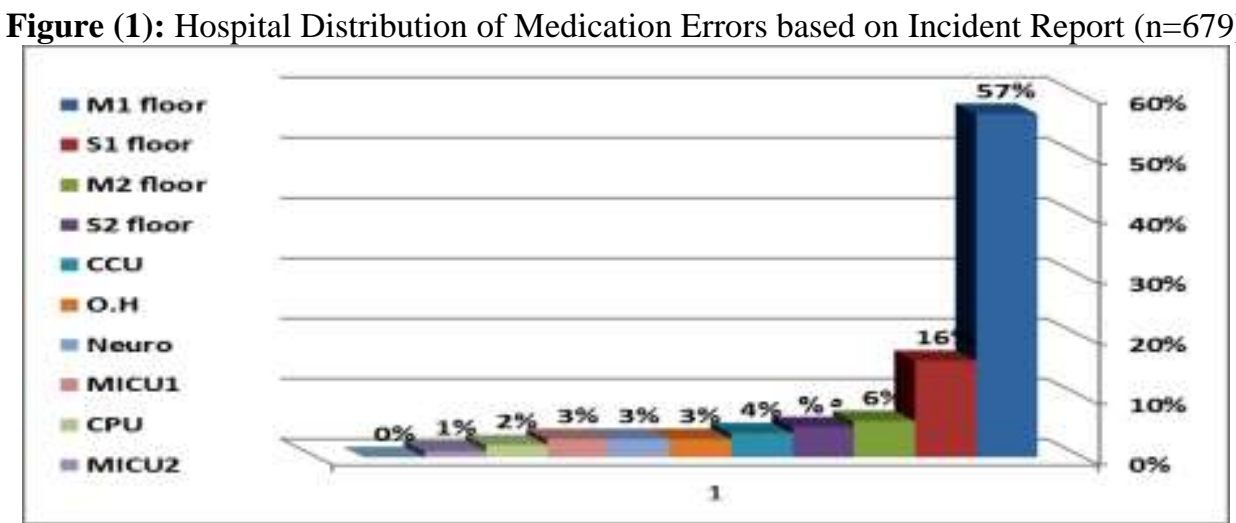

Figure (1) illustrates hospital distribution of medication errors based on incident report, it showed that the highest percentages of errors occurred in medical floor-1 (57\%) among all departments involved in the study.

Figure (2): Distribution of Medication Errors different causes as Presented by Incident Report (n=679)

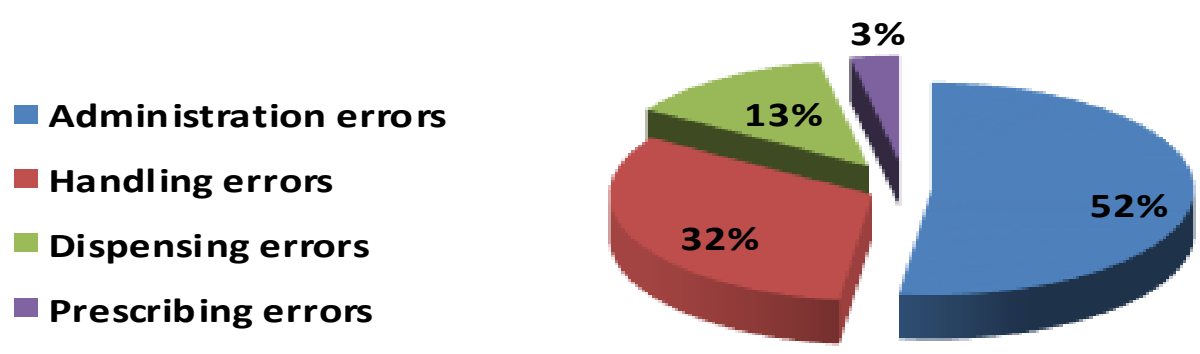

Figure (2) represents the first step of Pareto analysis which illustrates that administration errors had the highest percentage among the causes (52\%), while prescribing errors had the least (3\%).

Table (1): Frequency of medication errors $(n=679)$ rated in descending manner

\begin{tabular}{|c|c|c|}
\hline Items & Frequency & Percentage \\
\hline Administration errors & 355 & $52 \%$ \\
\hline Missed dose & 214 & $60.4 \%$ \\
\hline Delay administration & 60 & $16.6 \%$ \\
\hline Wrong frequency & 41 & $11.5 \%$ \\
\hline Wrong administration & 40 & $11.2 \%$ \\
\hline Handling errors & 219 & $32 \%$ \\
\hline Dispensing/receiving cycle & 200 & $29 \%$ \\
\hline Nurses carelessness & 19 & $2.7 \%$ \\
\hline Dispensing errors & 86 & $13 \%$ \\
\hline Dispensing wrong medication from pharmacy during rush hours & 60 & $9.0 \%$ \\
\hline Wrong dispense from porter during rush hours & 26 & $3.9 \%$ \\
\hline Prescribing errors & 19 & $3 \%$ \\
\hline Illegible hand writing & 19 & $3 \%$ \\
\hline Total & 679 & $100 \%$ \\
\hline
\end{tabular}

Table (1) demonstrates according to Pareto method, the percentage of medication errors problem which is rated in descending manner, as the finding indicated that missed dose $(60.4 \%)$ were the highest percentage in the category of administration errors, while, illegible hand writing were the least in the stage of prescribing errors.

Figure (3): Pareto Distribution of Medication Administration Errors 


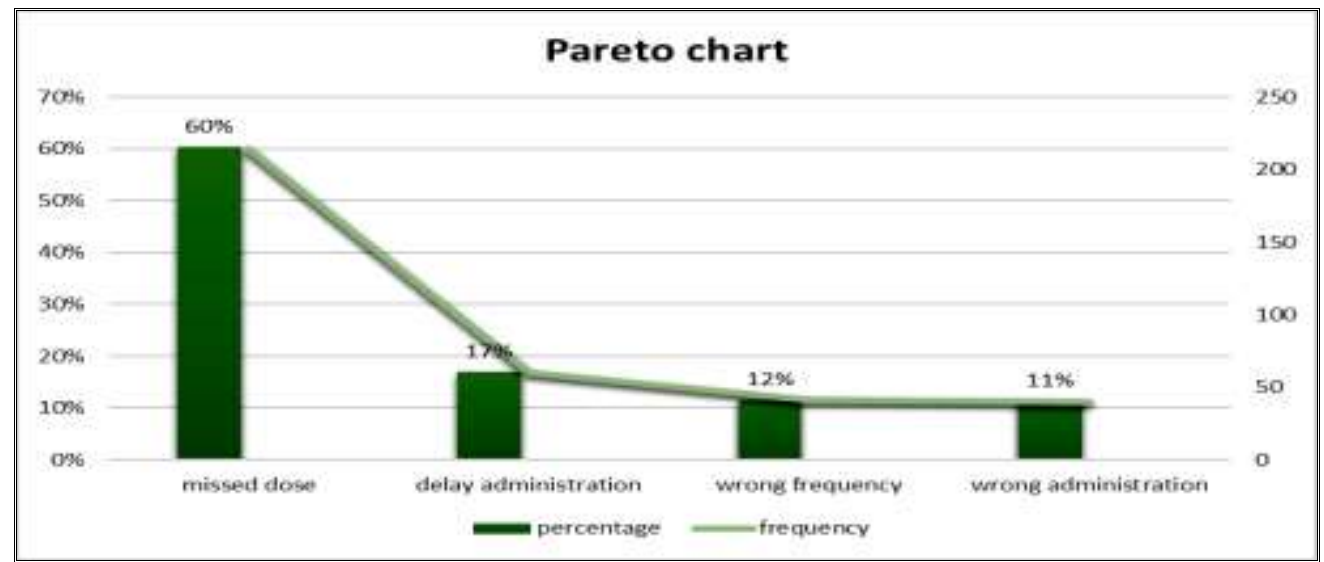

Figure (3) Pareto principal showed that, missed dose cause was the highest percentage $(60.4 \%)$ of the total medication administration errors while wrong administration was lowest percentage (11\%) happened during measurement phase.

Figure (4): The Possible Root Causes of Medication Administrations Errors (missed dose)

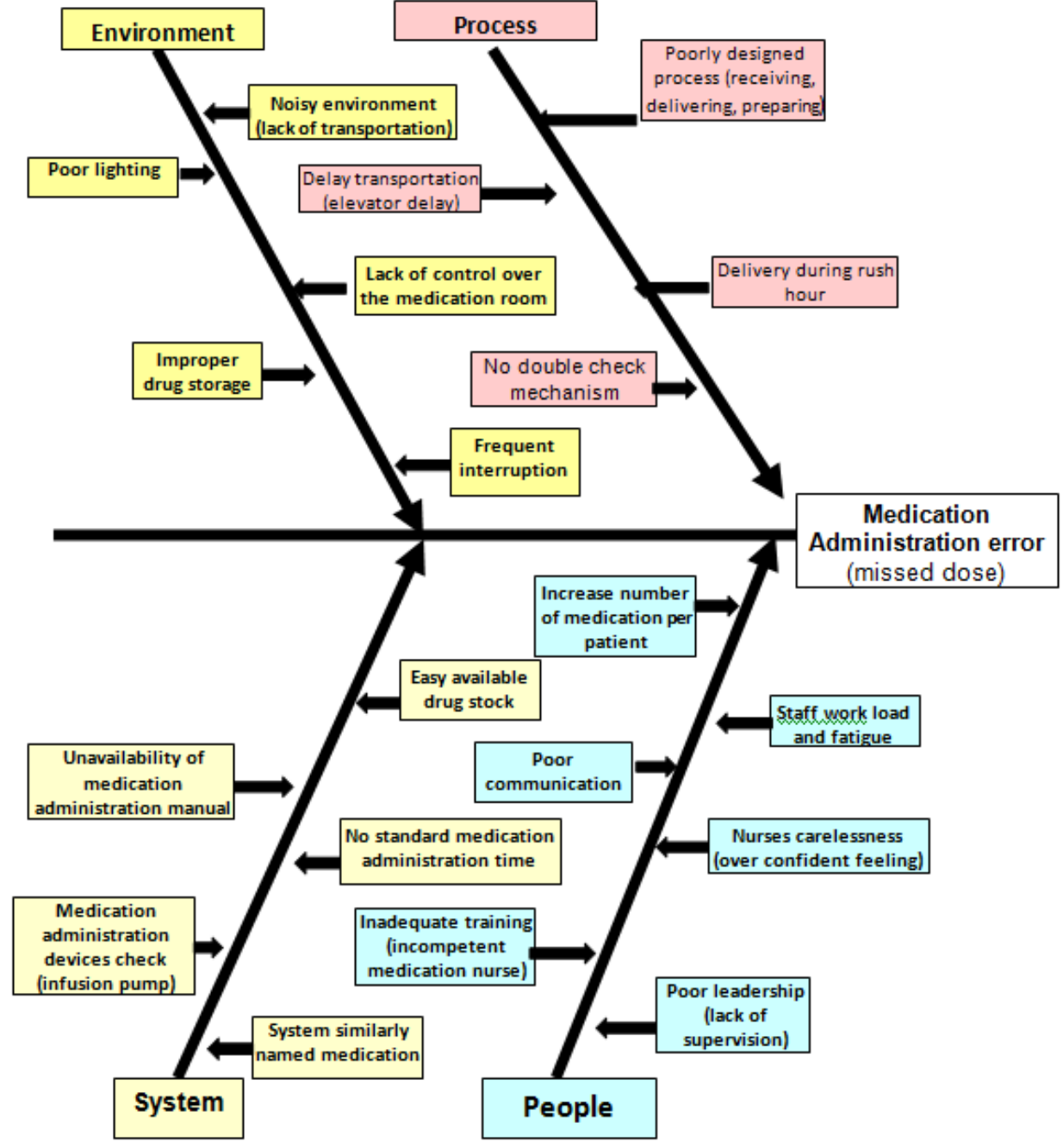

Figure (4) illustrate that according to process deficit, the possible causes found was poorly designed process (type of receiving/delivering/preparing distribution system), delay transportation (elevator delay), double check mechanism and delivery during rush hours. 
As regards to people deficit the possible root causes of medications administration errors were found in poor leadership, poor communication, inadequate training, work load, over confident feeling of the nurses (nurses' carelessness) and increase number of medications per patient.

The possible causes of environmental deficit were noisy environment, poor lightning-lack of control over the medication room, frequent interruption, lack of concentration and loose accountability.

Finally the system deficit represented as medication administration devices (infusion pump) check, no standard medication administration time, unavailability of medication administration manual, in spite of the hospital got JCI accreditation the medication administration manuals unavailable, and infusion pump not checked before use for its competency.

Table (2) Multi Voting Technique for Determining the Highest Scores of Possible Root Causes of Medication Administration Errors $(n=8)$

\begin{tabular}{|c|c|c|c|c|c|c|c|c|c|c|}
\hline \multicolumn{11}{|c|}{ Process deficit } \\
\hline \multicolumn{2}{|c|}{ Items } & \multicolumn{8}{|c|}{ Voting of (8) Team Members } & \multirow{2}{*}{$\begin{array}{r}\text { Score } \\
20\end{array}$} \\
\hline- & Poorly designed process (type of distribution system) & 3 & 3 & 2 & 1 & 3 & 3 & 2 & 3 & \\
\hline- & Delay pharmacy dispense & 1 & 2 & 1 & 1 & 2 & 0 & 1 & 1 & 9 \\
\hline- & Delivery during rush hours & 3 & 2 & 2 & 3 & 3 & 3 & 3 & 3 & 22 \\
\hline- & Delay transportation (extreme delay of elevator). & 2 & 1 & 3 & 2 & 2 & 1 & 1 & 0 & 12 \\
\hline \multicolumn{11}{|c|}{ People deficit } \\
\hline \multicolumn{2}{|c|}{ Items } & \multicolumn{8}{|c|}{ Voting of (8) Team Members } & Score \\
\hline- & Poor leader ship & 1 & 3 & 2 & 2 & 1 & 1 & 1 & 3 & 14 \\
\hline- & Inadequate training (incompetent nurse) & 0 & 0 & 1 & 0 & 2 & 1 & 1 & 0 & 5 \\
\hline- & Nurses carelessness & 1 & 0 & 0 & 3 & 0 & 1 & 1 & 0 & 6 \\
\hline- & Poor communication & 2 & 1 & 3 & 2 & 2 & 1 & 1 & 0 & 12 \\
\hline \multicolumn{11}{|c|}{ Practice deficit } \\
\hline \multicolumn{2}{|c|}{ Items } & \multicolumn{8}{|c|}{ Voting of (8) Team Members } & Score \\
\hline- & Incompetent medication nurse- nurses carelessness & 3 & 3 & 2 & 3 & 1 & 3 & 2 & 1 & 18 \\
\hline- & Inadequate assessment of patient condition & 1 & 0 & 0 & 3 & 0 & 1 & 1 & 0 & 6 \\
\hline- & No double check mechanism & 3 & 3 & 3 & 2 & 3 & 2 & 3 & 3 & 22 \\
\hline- & Delivery of medication during rush hours & 3 & 3 & 3 & 2 & 1 & 3 & 2 & 3 & 20 \\
\hline \multicolumn{11}{|c|}{ Environment deficit } \\
\hline \multicolumn{2}{|c|}{ Items } & \multicolumn{8}{|c|}{ Voting of (8) Team Members } & score \\
\hline- & Poor lightning & 2 & 2 & 1 & 0 & 1 & 3 & 1 & 1 & 11 \\
\hline- & Noisy environment & 3 & 3 & 3 & 1 & 2 & 2 & 3 & 3 & 20 \\
\hline- & No control over the medication room & 0 & 0 & 1 & 2 & 1 & 1 & 2 & 3 & 22 \\
\hline- & Frequent interruption & 0 & 1 & 1 & 2 & 1 & 0 & 2 & 2 & 10 \\
\hline
\end{tabular}

Table (2) illustrates the highest two score of possible root causes in each category. According to testing the possible causes of medication administration errors findings revealed that the highest two score of possible causes in the process deficit were; delivery during rush hours (scored 22), the second cause (scored 20) was poorly designed process (type of distributing system). Regarding people deficit the possible causes were: Poor leadership (scored 14) and poor communication (scored 12). As regard to the practice deficit the highest two scores of possible causes were no double check mechanism (scored 20) incompetent medication nurse (scored 18). Finally, related to environmental deficit it demonstrated that: no control over the medication room (scored 22) and noisy environment (scored 20).

Table (3) Final Possible Root causes of the Medication Administration Errors based on Fish Bone and Process Map Analysis

\begin{tabular}{|c|c|c|}
\hline \multicolumn{2}{|r|}{ Brainstorming } & Score \\
\hline \multirow{2}{*}{ Process deficit } & Poorly designed process (type of distribution system). & 20 \\
\hline & Delivery during rush hours. & 22 \\
\hline \multirow{2}{*}{ People } & Poor leadership skills. & 14 \\
\hline & Poor Communication. & 12 \\
\hline \multirow{2}{*}{ Practice deficit } & Incompetent medication nurse (years of experience). & 18 \\
\hline & No double check mechanism & 20 \\
\hline \multirow{3}{*}{ Environmental deficit } & Noisy environment. & 20 \\
\hline & No control over the medication room. & 22 \\
\hline & Process Map & \\
\hline \multirow{2}{*}{$\begin{array}{l}\text { Process deficit (Bottle- } \\
\text { neck) }\end{array}$} & $\begin{array}{l}\text { 9. Delay of the process and overload during the porter transport the drug from } \\
\text { process the pharmacy. }\end{array}$ & 20 \\
\hline & Process of receiving the drug from pharmacy. & 22 \\
\hline
\end{tabular}


Interdisciplinary Collaboration for Investigating Medication Errors Causes: Six Sigma

\begin{tabular}{|l|lll|}
\hline \multirow{2}{*}{ Constrains } & 11. & No multidisciplinary double check. & 14 \\
\cline { 2 - 4 } & 12. & Disconnection reporting system. & 12 \\
\hline \multirow{2}{*}{ Staff deficit } & 13. & Incompetent medication nurse. & 18 \\
\cline { 2 - 5 } & 14. & No double check mechanism. & 22 \\
\hline \multirow{2}{*}{ System deficit } & 15. & Errors (receiving \& checking) the drug from pharmacy. & 13 \\
\cline { 2 - 4 } & 16. & No control over the medication room & 20 \\
\hline
\end{tabular}

Table (3) indicates that there are 16 causes for the medication administration errors. Finding revealed that based on brainstorming the highest score in each component indicate the following errors occurrence (22 scores) happen in delivery during rush hours (process deficit), regarding (people) (14 scores) found in poor leadership skills, related to practice deficit ( 20 scores) found in no double check mechanism, as regards environmental deficit (22 scores) in no control over the medication room, in addition to the process mapping analysis, finding share the brainstorming analysis in its results, no double check mechanism and no control over the medication room. (22 and 20 scores), regarding process deficit, process of receiving the drug from pharmacy had (22 scores) and concerning constrains (14 scores) were found when no multidisciplinary double check occurred.

\section{III.Discussion}

Medication errors are often described as human errors in healthcare which attributed to many factors including level of knowledge and experience of health workers, workload and many other factors. Failure to improve could lead to patient harm which constitutes a medication error. Interdisciplinary collaboration team can learn much by closely analyzing and evaluating adverse events that lead to serious injury or death. The evaluation of such events enables them to improve the delivery of health care and reduce future mistakes. Small changes in healthcare providers can make a real difference in reducing harm to patients.

Regarding hospital distribution of medication errors based on incident report among the selected department, it was found that more than half of the study sample errors happened in medical floor, the fact which turns researchers' interest to investigate the causes and proceed in assessing the root cause analysis using multi quality techniques to overcome the problem. This is in disagreement with Brady (2009), who found that, about half of nurses are reserved about reporting medication errors because they fear disciplinary action. As reported by Agency for Healthcare Research and Quality (2014) whom found that, $48 \%$ of the study sample felt that person making the error was being written up instead of the actual problem being reported, and 35\% believe that these occurrences are recorded in their personnel files, in healthcare, a culture of fear and blame is associated with failure to report errors. A stigma is associated with making an error, and nurses perceive they will be a target for repercussions if they admit to making a mistake. On contrary of the study finding which indicates presence of effective reporting system and analyzing medication errors, nurses have a professional duty and moral obligation to report medication errors and presence of improvement culture inside the hospital

Concerning analysis of different errors of medication administration phases, which were categorized into four main items namely; prescribing, ordering, dispensing, distributing and administration, it was found that more than half of the incidence was in the administration errors phase; this is may be due to whenever there implementation phase errors arises due to diversity in experiences, educational level and work environment. This is in correspondence to Frith et al., (2012) in similar study who mentioned that the errors which reached the patient arise mainly from medication administration while the other reported errors across the different phases of medication delivery. Also Studies that examined the types of medication errors divided them in categories, according to the description of the event: omission error, wrong drug error, wrong patient error, wrong route error, wrong time error, wrong technique error, wrong dosage-form error and extra dose error. Thus, to avoid any type of medication error made by nurse, the implementation of preventive measures is undoubtedly beneficial. (Durham, 2015).

Regarding to pareto analysis, finding revealed that two third of the causes regarding medication errors phases was missed doses, this is may be due to lack of supervision, poor compliance with medication safety regulation and standards, there is no double check mechanism by two nurses, during rush hours the staff using medication of other patient drawer. This was in agreement with ISMP (2011), who states that, determination of causes of medication errors should be couple with assessment of error severity, while quality management processes should include programs to decrease the incidence, and established mechanisms for tracking drugs or drug classes that are involved in medication errors.

Concerning fish bone analysis, the study revealed that, there were four main root causes called the causal factors which were (process deficit, people deficit, environmental deficit and system deficit). The current study findings for the possible root causes of medication administration errors which result indicated that, missed dose was the highest percentage of the occurrence, it might be due to the process itself, poorly designed process receiving/delivering/preparing, delay transportation, delay pharmacy dispense, delivery during rush hours and no double check mechanism, this is with an agreement with Cadwell, (2008) who stated that, the vast 
majority of medication errors result from faulty systems and poorly designed processes versus poor practices or incompetent practitioners. Related to people deficit, findings revealed that poor leadership (lack of supervision, inadequate training, incompetent nurse, nurses carelessness, and poor communication, improper documentation, illegible handwriting, inadequate nurse-to-patient ratios can cause errors. These results are supported by Joint Commission, (2012) in a study of root causes analysis by event type; it was found that, human factors account for $73 \%$ of all medication errors. Also Choo et al., (2010) had found that, during medication administration, human errors are endorsed to the complex, multistep system processes established in the hospital.

Concerning environmental deficit, finding pinpoint toward noisy environment, lack of control over the medication room, poor lighting, improper drug storage, easy available floor stock and frequent interruption, increased number of medication per patient, and incompetent medication nurse, staff work load and fatigue, disconnected reporting system within the hospital, inadequate communication between healthcare provider. From the researchers point of view the correlation between system deficit and people deficit can increase the intensity of the problem. Health reform can be easily achieved if it is focused on the whole systems based on priorities of improvement. This finding is in consistent with Brady et al., (2009) who reported that, the lack of clinical decision supports, inadequate checks and balances, reliance on manual documentation, and insufficient information system infrastructures reduce the ability to interpret medication orders correctly. It is also supported by Dante et al., (2015) who showed that the organization of the medication administration process, healthcare providers' demands on nursing staff and the work environment are factors associated with medication administration interruptions. Regarding system deficit finding demonstrated that the highly score were in the item related to no control over the medication room. This may be due to nurse's heavy workload, excessive paper work and poor supervision which contribute to increase errors. This finding was in concurrence with Choo et al., (2013) who mentioned that system corruption including different kinds of distractions can affect safe medication management because they impair nurses' level of concentration and increase their work pressure.

\section{Conclusion}

The aim of this study was to assess hospital team in tracing medication errors causes using incident report and investigate medication errors through using six sigma methodologies among nursing staff. The current study had accepted the research hypothesis by evidence that, interdisciplinary collaboration team by using Six sigma can identify the root cause of medication administration errors inside the hospital which is missed dose in addition to the process itself as well as the human factors were the major causes. An additional training program needed for interdisciplinary teams for continued project work to improve patient safety and build on to complete the Six Sigma phases to assist in error reduction.

\section{Recommendations}

Based on the study finding, it is recommended the following:

1. Quality improvement advancing program and peer review should be implemented to assist in identifying causes of errors and eradicating its recurrence.

2. All managerial categories should be engaged in the improvement process to get their commitment to quality standards.

3. Training on improvement process should be provided to increase awareness and force the internal auditing at unit level.

4. Medication administration training program should be conducted for nurses, asserted of performance reporting system, and encouraged the use of root-cause analysis in all work process to contribute improvement environment.

\section{References}

[1]. Parand Anam, Dopson Sue, Renz Anna, Vincent Charles (2014): The role of hospital managers in qualityand patient safety: a systematic review; BMJ Open 4:e005055. doi:10.1136/bmjopen-2014-005055

[2]. Bender, M.; Connelly, C.D.; \& Brown, C. (2013): Interdisciplinary collaboration: The role of the clinical nurse leader. Journal of Nursing Management, 21, 165-174.

[3]. Taghizadegan, S. (2011): Statistical Process Control. Journal of quality Practice Management. 26: 12-16.

[4]. National Coordinating Council for Medication Error Reporting and Prevention, (2015): About medication errors: What is a medication error? Available at: http://www.nccmerp.org/about-medication-errors.

[5]. Bush, K. (2010): Fixing the System: Practical Examples of Patient Safety and Learning From Adverse Events in Respiratory Therapy. Canadian Journal of Respiratory Therapy, 46(4), 6-12.

[6]. Hewitt, T. A. (2013): Incident Reporting Systems - The Hidden Story. The Journal of the Safety and Reliability Society, 33(2), 1328.

[7]. Bisgaard, S.; Hoerl, R. W. \& Snee (2014): Improving healthcare processes with Six Sigma. Quality Management in Healthcare journal 16: 28-33.

[8]. Esmail, A. (2011): Patient safety in your practice. Pulse; 71: 3, 22-23, retrieved from: Using fishbone analysis to investigate problems, Nursing Times 16.04.13/ Vol 109 No 15/ www.nursingtimes.net. 
[9]. Jusko, J. (2012): A look at six sigma, international journal of six sigma and competitive advantage. 8(5), 15-21.

[10]. Brady, A.M.; Malone, A.M.; Fleming, S.A. (2009): literature review of the individual and systems factors that contribute to medication errors in nursing practice. J Nurs Manag. 2009;17(6):679-697.

[11]. Agency for Healthcare Research and Quality (2014): Hospital Survey on Patient Safety Culture: User Comparative Database Report: Chart 5-2. Item-level average percent positive response-2014 database hospitals. 2014. http://www. ahrq.gov/professionals/quality-patient-safety/patientsafetyculture/hospital/2014/hosp14chart5-2.html.

[12]. Frith, K.H.; Anderson, E.F.; Tseng, F. \& Fong, E.A. (2012): Nurse staffing is an important strategy to prevent medication error in community hospitals. American Nurses Association. 30(5): 288-294. [Online]. Available:

[13]. http://www.nursingworld.org/MainMenuCategories/ThePracticeofProfessionalNursing/Nur seStaffing/2014-Nurse-StaffingUpdated-Literature-Review.pdf [2015, May 13].

[14]. Durham Barbara, (2015): the nurse's role in medication safety ; Wolters Kluwer Health, Inc; online exclusive

[15]. (ISMP) - Institute of safe medication practice (2011): ISMP's list of high alert medication. Retrieved October 24, 2009 from http://ismp.org/Tools/highalertmedication.pdf.

[16]. Cadwell, (2008): Pediatric medication safety in the emergency department. Journal of emergency nursing, 34(7). 375-377.

[17]. The Joint Commission (2012): Sentinel event data: root causes by event type $2004-2012$. http://www.jointcommission.org/assets/1/18/Root_Causes_Event_Type_04_4Q2012.pdf.

[18]. Choo, J.; Hutchinson, A.; Bucknall, T. (2010): Nurses' role in medication safety. J Nurs Manag.;18(7):853-861.

[19]. Dante, A.; Andrigo, I.; Barone, F. et al. (2015): Occurrence and duration of interruptions during nurses' work in surgical wards. J Nurs Care Qual 2015;31:174-82.

Choo, J.; Johnston, L. \& Manias, E. (2013): Nurses' medication administration practices at two Singaporean acute care hospitals. Nursing and Health Sciences 15(1), 101-108. 